EGU21-12292

https://doi.org/10.5194/egusphere-egu21-12292

EGU General Assembly 2021

(c) Author(s) 2021. This work is distributed under

the Creative Commons Attribution 4.0 License.

\title{
Autocorrelation of the ground vibration recorded by the SEIS- InSight seismometer on Mars for imaging and monitoring applications
}

Nicolas Compaire ${ }^{1}$, Ludovic Margerin ${ }^{2}$, Raphaël F. Garcia ${ }^{1,2}$, Marie Calvet ${ }^{2}$, Baptiste Pinot ${ }^{1}$, Guénolé Orhand-Mainsant ${ }^{1}$, Doyeon Kim ${ }^{8}$, Vedran Lekic ${ }^{8}$, Benoit Tauzin ${ }^{10,11}$, Martin Schimmel ${ }^{9}$, Eléonore Stutzmann ${ }^{3}$, Brigitte Knapmeyer-Endrun ${ }^{4}$, Philippe Lognonné ${ }^{3}$, William T. Pike ${ }^{5}$, Nicholas Schmerr ${ }^{8}$, Laurent Gizon ${ }^{7}$, and Bruce Banerdt ${ }^{6}$

${ }^{1}$ Université Fédérale de Toulouse Midi-Pyrénées, ISAE-Supaero, DEOS (Département Electronique, Optronique et Signal), Toulouse, France (nicolas.compaire@hotmail.fr)

${ }^{2}$ Institut de Recherche en Astrophysique et Planétologie, Université Toulouse III Paul Sabatier, CNRS, CNES, 14 Av. E. Belin, 31400, Toulouse, France.

${ }^{3}$ Université de Paris, Institut de physique du globe de Paris, CNRS, F-75005 Paris, France.

${ }^{4}$ Bensberg Observatory, University of Cologne, Vinzenz-Pallotti-Str. 26, 51429 Bergisch Gladbach, Germany.

${ }^{5}$ Department of Electrical and Electronic Engineering, Imperial College London, South Kensington Campus, London, SW7

2AZ, United Kingdom.

${ }^{6}$ Jet Propulsion Laboratory, California Institute of Technology, Pasadena, CA 91109, USA.

${ }^{7}$ Max Planck Institute for Solar System Research, Justus-von-Liebig-Weg 3, 37077 Göttingen, Germany.

${ }^{8}$ University of Maryland, College Park, Department of Geology, 8000 Regents Dr., College Park, MD, 20742, USA.

${ }^{9}$ Institute of Earth Sciences Jaume Almera - CSIC, Barcelona, Spain.

${ }^{10}$ Université de Lyon, Université Claude Bernard Lyon 1, ENS, CNRS, Laboratoire de Géologie de Lyon : Terre, Planètes,

Environnement, 69622 Villeurbanne, France.

${ }^{11}$ Research School of Earth Sciences, The Australian National University, Canberra, Australian Capital Territory 0200,

Australia.

Since early February 2019, the SEIS seismometer deployed at the surface of Mars in the framework of the NASA-InSight mission has been continuously recording the ground motion at Elysium Planitia. In this work, we take advantage of this exceptional dataset to put constraints on the crustal properties of Mars using seismic interferometry (SI). This method use the seismic waves, either from background vibrations of the planet or from quakes, that are scattered in the medium in order to recover the ground response between two seismic sensors. Applying the principles of $\mathrm{SI}$ to the single-station configuration of SEIS, we compute, for each Sol (martian day) and each local hour, all the components of the time-domain autocorrelation tensor of random ambient vibrations in various frequency bands. A similar computation is performed on the diffuse waveforms generated by more than a hundred Marsquakes. For imaging application a careful signal-to-noise ratio analysis and an inter-comparison between the two datasets are applied. These analyses suggest that the reconstructed ground responses are most reliable in a relatively narrow frequency band around $2.4 \mathrm{~Hz}$, where an amplification of both ambient vibrations and seismic events is observed. The average Auto-Correlation Functions (ACFs) from both ambient vibrations 
and seismic events contain well identifiable seismic arrivals, that are very consistent between the two datasets. We interpret the vertical and horizontal ACFs as the ground reflection response below InSight for the compressional waves and the shear waves respectively. We propose a simple stratified velocity model of the crust, which is most compatible with the arrival times of the detected phases, as well as with previous seismological studies of the SEIS record. The hourly computation of the ACFs over one martian year also allows us to study the diurnal and seasonal variations of the reconstructed ground response with a technique call Passive Image Interferometry (PII). In this study we present measurements of the relative stretching coefficient between consecutive ACF waveforms and discuss the potential origins of the observed temporal variations. 